\title{
Characterization of a Novel Structural Feature termed Pseudovacuole Located in the Front of Walker Carcinosarcoma Cells
}

\author{
D. Vanhecke, Eggli, P., Graber, W., Keller, HU., Studer, D.
}

Abteilung für Topographische Anatomie und Neuroanatomie, Institute for Anatomy, University of Bern, Baltzerstrasse 2, 3000 Bern 9, Switzerland

The unraveling of cellular motility at sub-cellular level started with the first cellular locomotion model by Mast [1]. Almost 80 years later, large gaps are still present in the understanding of this crucial process. A paper published by Keller et al. in 2002 [2] showed that destroying the frontal actin as well as microtubules is not influencing the locomotion process in Walker carcinosarcoma cells. In the search for other factors, we found a structure with a vacuole-like appearance. We termed it pseudovacuole, because a membrane is never surrounding it.

In contrast to the cytoplasm of the cell body - which has a coarse-grained aspect due to the presence of organelles - the frontal part of Walker carcinosarcoma cells has a characteristic hyaline appearance. Differential interference contrast microscopic observations show that the pseudovacuole occurs in the hyaline part of the cell, and appears as a homogeneously filled, round, ovoid or sausage-like structure sharply bordered from the surrounding cytoplasm (Fig. 1, 2a). A survey of 414 cells demonstrated that the majority of the cells $(62.1 \%)$ possessed such a pseudovacuole. Mostly one pseudovacuole, rarely more are present per cell. Three fluorescent markers revealed basic information: a lipid marker $\left(\mathrm{DiIC}_{18}\right)$, a nucleic acid marker (SYTO17) and a protein marker (CFDA SE, a lysine marker). The corresponding fluorescence distributions are depicted in fig. 2. The lipid marker and the nucleic acid marker both are labeling specific regions in the cell, but both are lacking in the pseudovacuole (Fig $2 \mathrm{~b}$ and c). The lysine marker however shows the presence of lysine-containing proteins in almost the whole cell, but the pseudovacuole exhibits a much higher signal (Fig 2d). Ultrastructural details were described by applying transmission electron microscopic (TEM) analysis. However, conventional fixation techniques were not sufficiently preserving the content and the morphology of the pseudovacuoles (Fig. 3a). Using cryomethods (high pressure freezing followed by freeze substitution), a better preservation of the pseudovacuoles was achieved. Artifact formation was kept to a minimum by fast sampling [3]: less than 1 minute was needed to transfer the cells from a flask with cell suspension at $37^{\circ} \mathrm{C}$ until the cells were high pressure frozen in the Leica EMPACT at $-196^{\circ} \mathrm{C}$ [4]. The use of cryomethods retained the content of the pseudovacuoles and the acquired TEM data show the presence of inclusions that correspond in size, shape and location to pseudovacuoles (Fig. 3b). A loosely ordered arrangement - absent in chemical fixed samples - makes up the content of the inclusion (Fig. 3c). Organelles and ribosomes are absent in the inclusions (Fig. 3c and d), and a surrounding membrane could never be revealed (Fig. 3d).

We think that the pseudovacuole may act as an osmotic regulator responsible for changing the hydrostatic pressure, which leads - in cooperation with actomyosin depending contraction in the back - to frontal protrusions.

[1] Mast, S.O. (1926) J. Morph. Phys. 41(2): 247-424. 
[2] H.U. Keller et al. (2002) Cell mot. cytoskeleton 53:189-202.

[3] D. Vanhecke et al. (2003) J. Microsc. 212(1):3-12.

[4] D. Studer et al. (2001) J. Microsc. 203(3):285-94.

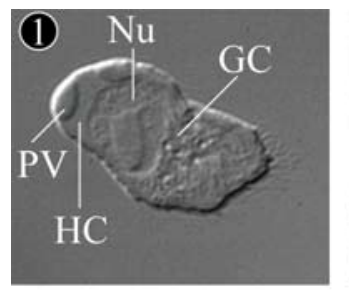

(3)
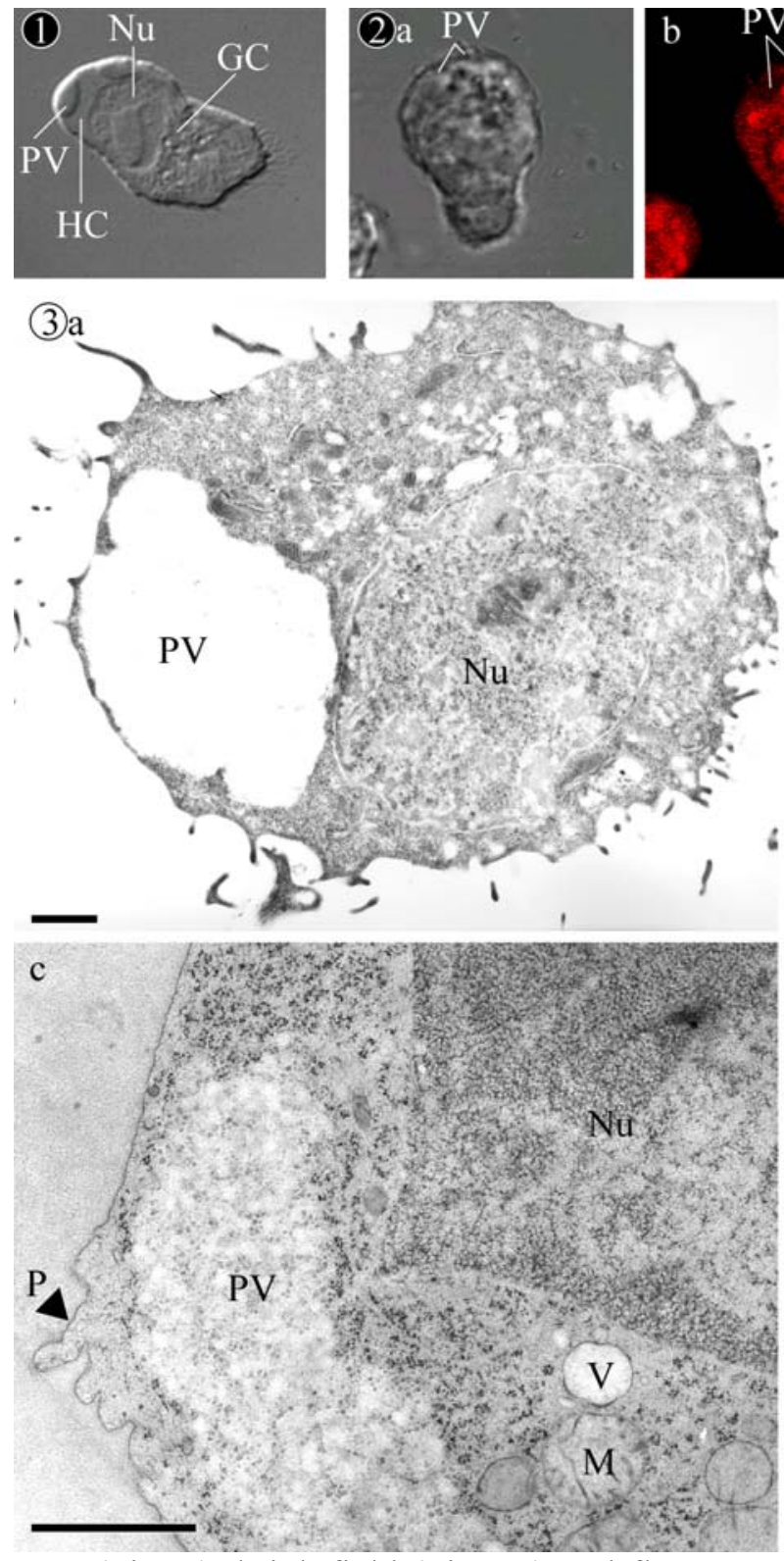

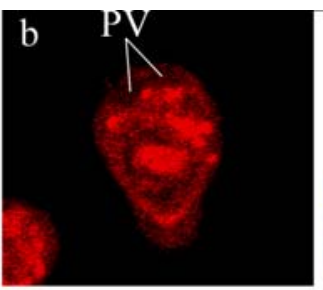

b
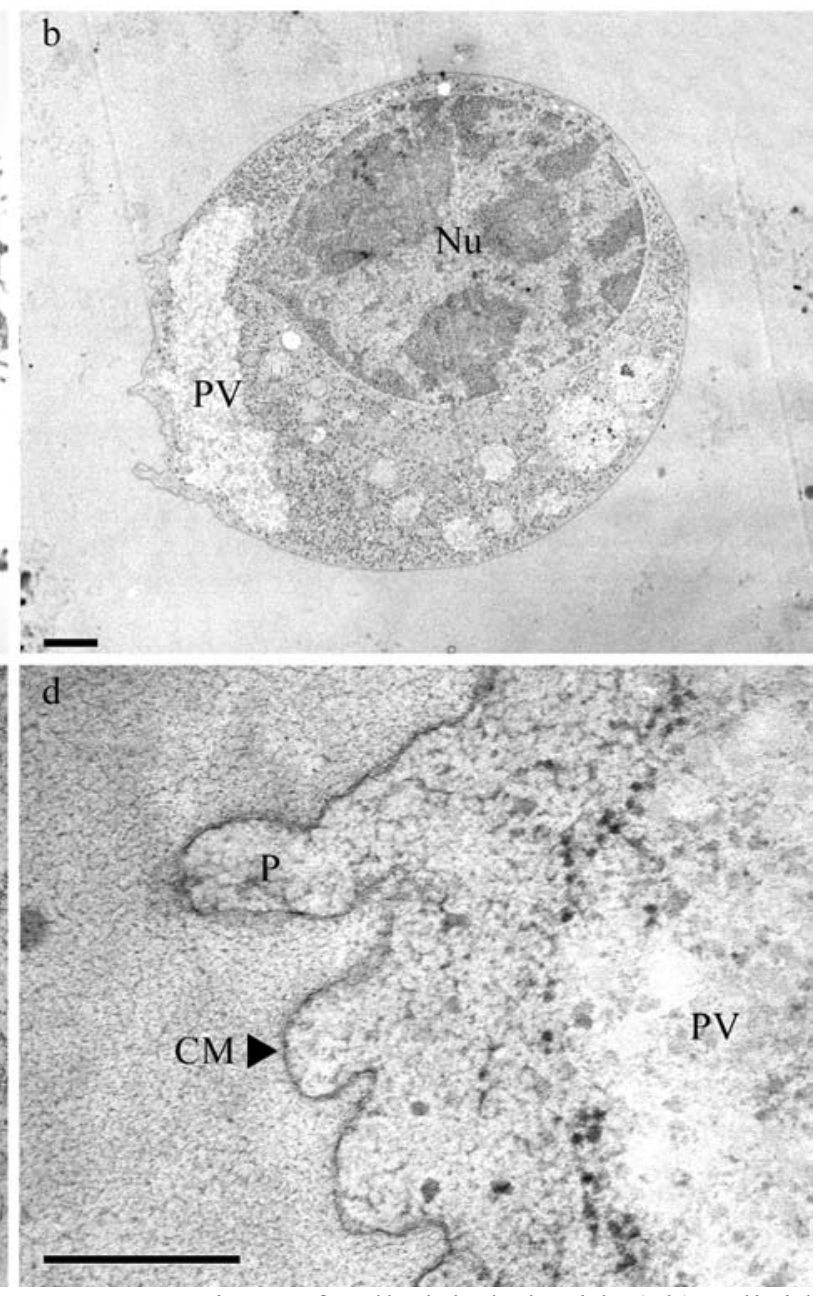

DIC (Fig. 1), brightfield (Fig. 2a) and fluorescent representations of cells labeled with (2b) a lipid marker $\left(\right.$ DiIC $\left._{18}\right)$, (2c) a nucleic acid dye (SYTO17) and (2d) a lysine marker (CFDA SE). GC= granular cytoplasm, $\mathrm{HC}=$ hyaline cytoplasm, $\mathrm{PV}=$ pseudovacuole. Fig 3. Transmission electron micrographs of a chemically (a) and a cryofixed Walker carcinosarcoma cell (b, c and d), CM=cell membrane, $\mathrm{M}=$ mitochondrion, $\mathrm{Nu}=$ Nucleus, $\mathrm{P}=$ protrusion, $\mathrm{PV}=$ pseudovacuole, $\mathrm{V}=$ vesicle. $\mathrm{Bar}=2$ $\mu \mathrm{m}$, except Fig. 2d: Bar=500nm. 